\title{
Micro-mechanics of Foam using Unit Cell (Closed Cell) Approach
}

\author{
G. Venkatachalam*
}

School of Mechanical and Building Sciences, VIT University, Vellore-632014, India; g.venkatachalam@vit.ac.in

\begin{abstract}
Because of its wide range of properties, usages of foam in various applications are enormous, which normally cannot be met by fully dense solids. The foam is considered as a periodic array of unit cells. The unit cell taken for the analysis is tetrakaidecahedron. The objective of the present work is to understand the micromechanics of foam deformation and prediction of elastic properties by numerical simulation using FEA software ABAQUS for closed cell foam. These properties are compared with those available in literature. Translation symmetry of the unit cell alone is sufficient to get the property of the entire foam. The periodic boundary conditions are imposed on the unit cell. The values obtained from the present analysis give better results since the periodicity is maintained. The Young's modulus and shear modulus are the increasing functions of relative density while the Poisson's ratio is a decreasing function.
\end{abstract}

Keywords: Tetrakaidecahedron, Closed Cell, Translation Symmetry, Foam, Elastic Properties.

\section{Introduction}

Foams are solids made up of an interconnected network of solid struts or plates, which form the edges and faces of cells. The cells are of polyhedral shape and pack in three dimensions to fill space. The density of foam is much lower than that of solid material constituting its struts or plates. This low density of foam permits the design of light, stiff components such as sandwich panels and large portable structures, and of floatations of all sorts. Also, the low strengths and large compressive strains make foam attractive for energy absorbing applications. The mechanical behaviour of foam can either be described by macro-mechanical phenomenological constitutive laws or by micro-mechanics. Micro-mechanics tries to relate the behaviour of foam in terms of deformation behaviour occurring at single cell level. The later approach is attempted in the present work. The deformations at the cell level are calculated using a unit cell approach and periodic boundary conditions in combination with finite element methods. The elastic properties such as E, $v$ and $\mathrm{G}$ are determined using this method. In it, the properties of foam are related to its structure and to the properties of the material of which the cell walls and edges are made. The present work is focused on understanding the mechanical behaviour of foams using numerical simulations. For closed cell foams, the cell faces are considered as plates. The results obtained by our analysis are then compared with those available in literature.

\section{Introduction to Unit Cell Model}

The single most important structural characteristics of a cellular solid is its relative density $\rho^{\prime} / \rho_{s_{s}}$ (the density, $\rho^{\prime}$, of the foam divided by that of the solid of which it is made, $\left.\rho_{s}\right)$. The properties of cellular solids directly depend on the shape and structure of the cells and hence the structure of cells is important to us. When the cells are equiaxed, the properties are isotropic, but when the cells are even slightly elongated or flattened the properties depend on direction, often strongly so. A single tetrakaidecahedron cell isolated

* Corresponding author:

G. Venkatachalam (g.venkatachalam@vit.ac.in) 


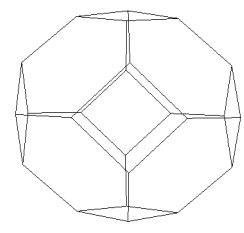

Figure 1. Tetrakaidecahedron Unit cell.

from the foam material is used as a representative volume element (RVE), which is of idealized nature. The tetrakaidecahedron is a popular choice because it has a maximum average number of edges per face and of faces per cell. The Figure 1 shows the structure of tetrakaidecahedron, a 14-sided polyhedron generated by uniformly truncating the six corners of an octahedron. Also, the tetrakaidecahedron cell is known to be the only polyhedron that can pack with identical units to fill space and nearly minimizes the surface energy [4].

The book by Gibson and Ashby [1] on cellular solids discusses strength of materials based mechanics of deformation of foams and gives the necessary equations, for various moduli \& Poisson's ratios as functions of relative density (R). Li et al. [2] studied the micromechanics model for three dimensional open cell (carbon) foams and developed relations for mechanical properties using an energy method based on Castigliano's second theorem. Roy et al. [4] developed a micromechanics model for threedimensional open-cell foams by using tetrakaidecahedral unit cells. Li and Wongsto [3] established the periodic boundary conditions using unit cells for micromechanical analysis of particle-reinforced composites. Venkatachalam [5] developed a unit cell (open cell) model using tetrakaidecahedron by applying periodic boundary conditions.

\section{Finite Element Formulation}

Having established the unit cell, viz. the tetrakaidecahedron that will generate the complete foam, we establish the periodic boundary conditions. The foam material is considered as a small repetitive unit cell element, called representative volume element.

\subsection{Closed Cell Foam}

The faces of the cell are considered as a plate. Mindlin shell elements (based on Timoshenco plate theory - S4 in ABAQUS) with six degrees of freedoms (d.o.f.s) at each node are used. The six d.o.f.s are $\mathrm{u}, \mathrm{v}, \mathrm{w}, \theta_{\mathrm{x}}, \theta_{\mathrm{y}}$ and $\theta_{\mathrm{z}}$. The close cell discretized in to finite elements is shown in Figure 2. There are 1066 nodes and 1064 elements used in the analysis. After applying the boundary conditions [3], we can get the desired strain in the desired direction by giving the suitable values of displacement. For example, we want to have strain only in one direction $\left(\varepsilon_{1}\right)$ and all other strains are zero, which are shown in Figure 3. Displacements are there in II and III directions but relative displacements are zero and hence strains in II and III directions are zero.

\section{Results and Discussions}

The current unit cell model considered here has a cubic symmetry and hence the constants E, G and $v$ are independent of each other. The foam material that we have taken for the analysis is of polystyrene. The analysis is carried out by using the commercial FE analysis software ABAQUS.

The reaction forces in the faces perpendicular to $\mathrm{x}, \mathrm{y}$ and $\mathrm{z}$ are obtained and from these, the required stresses

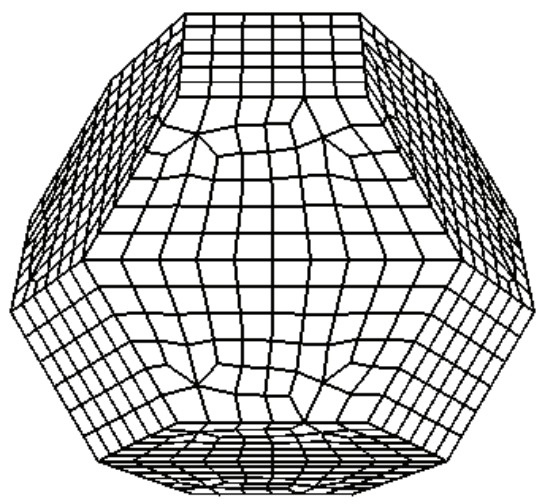

Figure 2. Closed cell with finite elements.

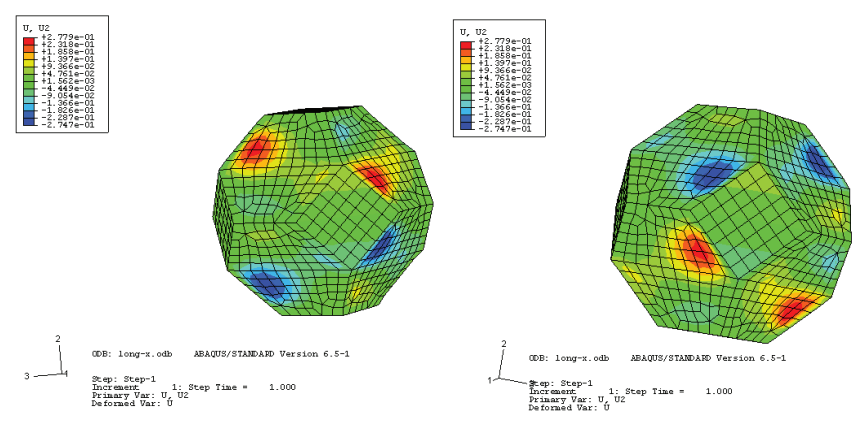

Figure 3. Closed cell showing deformations in II direction. 
are calculated. The relative density of the foam is taken in the range of 0.01 to 0.1 . The predicted values of $E, v$ and $\mathrm{G}$ varying with $\mathrm{R}$ are plotted in Figures. (4, 5 and 6). The values of elastic properties obtained by the current model (Unit Cell-Closed) are compared with those of the model proposed by Roy et al. [4] and Venkatachalam [5] as they have shown close agreement with the values available in literature. Figures 4 and 6 compare respectively, the Young's modulus (E) and shear modulus (G) predicted by the present model with those of Roy et al. [4] and Venkatachalam [5]. The values are nearer to those of [4] and [5] for almost all values of relative density. The rate of increase in $\mathrm{E}$ and $\mathrm{G}$ at higher relative densities for closed cell model is higher than that of Roy et al. [4] and Venkatachalam [5]. Figure 5 illustrates that $v$ decreases as $\mathrm{R}$ increases. The rate of decrease in Poisson's ratio at higher relative densities predicted by the open cell model and closed cell model is little higher than the Roy et al. [4]. Poisson's ratio is close to 0.5 as relative density approaches zero. This trend is same as that predicted by Roy et al. [4] and Venkatachalam [5]. Also, values of $v$ predicted by the current model are close to the values suggested by Gibson and Ashby [1].
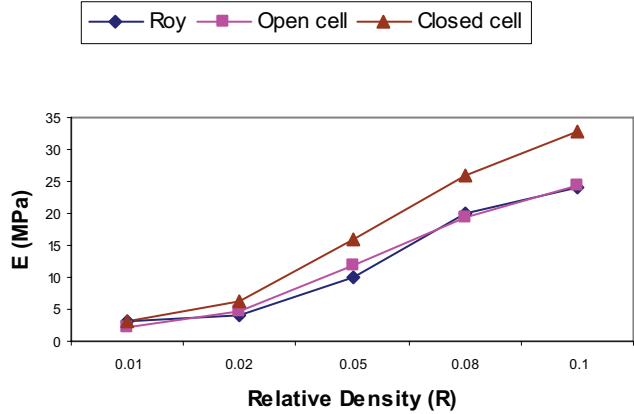

Figure 4. Young's modulus of foam as function of R.
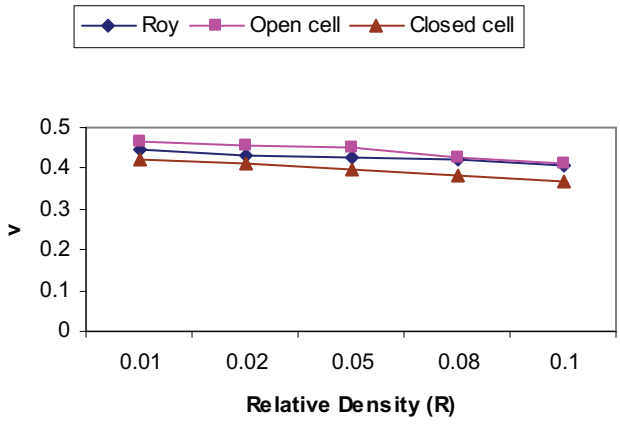

Figure 5. Poisson's Ration of foam as function of R.
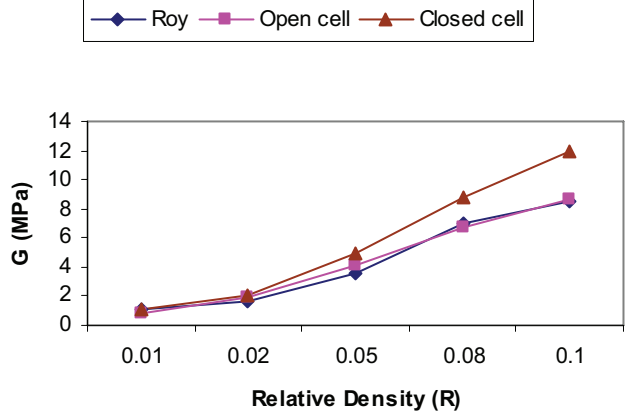

Figure 6. Shear modulus of foam as function of R.

It is found that the behaviour of foam in the linear elastic domain is same for both tension and compression are same.

\section{Conclusion}

A micro-mechanical model for predicting the effective elastic properties of foam is developed using a tetrakaidecahedral unit cell. The FE analysis is carried out using translational symmetry on closed cells. The periodic boundary conditions are imposed on the unit cell, as the unit cell is a repetitive element. The results are compared with those of analysis reported by others. The values obtained from the present analysis give better results since the periodicity is maintained. The Young's modulus and shear modulus are the increasing functions of relative density while the Poisson's ratio is a decreasing function.

\section{Reference}

1. Gibson L J, and Ashby M F (1988). Cellular solids- structure and properties, Chapter 5, Pergamon Press, Oxford, $175-231$.

2. Li K, Gao X L et al. (2003). Micromechanics model for three dimensional open cell foams using tetrakaidecaherdral unit cell and Castigliano's second theorem, Composite Science and Technology, vol 63(12), 1769-1781.

3. Li S, and Wongsto A (2004). Unit cells for micromechanical analysis of particle-reinforced composites, Mechanics of Materials, vol 36(7), 543-572.

4. Roy A K, Gao X L et al. (2005). Micromechanical modeling of three-dimensional open-cell foams using the matrix method for spatial frames, Composite part A: Engineering, vol 36, 249-262.

5. Venkatachalam G (2008). Micro-Mechanics of Foam using Unit Cell Approach, Journal of The Institution of Engineers (India)-AS, vol 89, 29-31. 